\title{
Prevalencia de patrones electrocardiográficos asociados a muerte súbita en la población española de 40 años o más: resultados del estudio OFRECE
}

\author{
Paula Awamleh García ${ }^{a}$, Joaquín Jesús Alonso Martín ${ }^{a}$, Catherine Graupner \\ Abad $^{\mathrm{b}}$, Rosa María Jiménez Hernández ${ }^{\mathrm{b}}$, Alejandro Curcio Ruigómez ${ }^{\mathrm{b}}$, Pedro \\ Talavera Calle ${ }^{\mathrm{b}}$, Carmen Cristóbal Varela ${ }^{\mathrm{b}}$, José Serrano Antolín ${ }^{\mathrm{b}}$, Javier Muñiz ${ }^{\mathrm{c}}$, \\ Juan José Gómez Doblas ${ }^{\mathrm{d}}$, Eulalia Roig ${ }^{\mathrm{e}}$
}

\footnotetext{
a Servicio de Cardiología, Hospital Universitario de Getafe, Getafe, Madrid, España

${ }^{\mathrm{b}}$ Servicio de Cardiología, Hospital Universitario de Fuenlabrada, Fuenlabrada, Madrid, España

${ }^{c}$ Instituto Universitario de Ciencias de la Salud e Instituto de Investigación Biomédica de A Coruña (INIBIC), Universidad de A Coruña, A Coruña, España

${ }^{\mathrm{d}}$ Servicio de Cardiología, Hospital Clínico Universitario Virgen de la Victoria, Málaga, España

${ }^{\text {e }}$ Servicio de Cardiología, Hospital de la Santa Creu i Sant Pau, Barcelona, España
}

\begin{abstract}
Resumen
Introducción y objetivos. Hay patrones electrocardiográficos asociados a mayor riesgo de muerte súbita por arritmias ventriculares. En España no existe información acerca de su prevalencia en la población. El objetivo es estudiar la prevalencia de estos patrones, así como los factores clinicoepidemiológicos asociados a su presencia.

Métodos. Subanálisis del estudio OFRECE en el que se estudió la prevalencia de patrones electrocardiográficos de síndrome de Brugada o anomalías del intervalo QT en una muestra representativa de la población española $\geq 40$ años. Se dispuso de datos clínicos y electrocardiogramas de todos los participantes. Los electrocardiogramas fueron evaluados de forma independiente por 2 cardiólogos y, en caso de desacuerdo, se consultó con un tercero. Se analizaron las prevalencias ponderadas y los factores clínicos asociados a patrones tipo Brugada o a anomalías del segmento QT.

Resultados. Se evaluó a 8.343 individuos (59,2 años, 52,4\% mujeres) y se detectaron 12 casos de patrón Brugada (tipo 1, 2 casos; tipo 2, 10 casos; prevalencia ponderada, 0,13\%). Para el análisis del QT corregido (QTc) se excluyó a los participantes con bloqueo de rama izquierda o ritmos no sinusales. Las prevalencias ponderadas fueron: QTc corto (<340 ms) 0,18\%, QTc borderline (441-469 ms) 8,33\%, QTc largo (criterio $\geq$ $470 \mathrm{~ms}$ ) $1,01 \%$ y QTc largo (criterio $\geq 480 \mathrm{~ms}$ ) $0,42 \%$.

Conclusiones. El $0,6-1,1 \%$ de la población española de edad $\geq 40$ años presenta un patrón electrocardiográfico de riesgo de muerte súbita (síndrome de Brugada, QT largo o QT corto).
\end{abstract}

Palabras clave

Muerte súbita; Epidemiología; Síndrome de QT largo; Taquiarritmias 


\section{INTRODUCCIÓN}

En los últimos 20 años se han definido síndromes clinicoelectrocardiográficos asociados con mayor riesgo de muerte súbita (MS) por arritmias ventriculares como el síndrome de Brugada ${ }^{1,2,3,}$ ${ }^{4}$ o el síndrome de QT corto ${ }^{5,6}$ y se han tipificado mejor otros ya conocidos, como el síndrome de QT $\operatorname{largo}{ }^{7,8}$. Estas enfermedades pueden cursar de forma asintomática durante años y su prevalencia en diversos estudios europeos es variable: entre 0 y $6 \%{ }^{9,10}$ para los patrones de Brugada y 0 y $0,5 \%$ para el QT corto ${ }^{11}$. Los estudios de prevalencia del QT largo en la población general son muy heterogéneos (diferentes poblaciones, distintos puntos de corte). El síndrome de QT largo es el trastorno arrítmico asociado a MS más frecuente en la práctica clínica $(35,6 \%)$ seguido del síndrome de Brugada $(32,1 \%)^{12}$. El objetivo de este estudio es estimar, en una muestra representativa de la población española, la prevalencia de patrones electrocardiográficos compatibles con Brugada tipos 1 y 2, QT largo y QT corto.

\section{MÉTODOS}

Este trabajo es un subestudio del proyecto OFRECE (Observación de FibRilación y Enfermedad Coronaria en España), aprobado por el Comité Ético de Investigación Clínica del Hospital Universitario de Basurto y por otros comités hospitalarios o regionales. Su objetivo era conocer la prevalencia de fibrilación auricular (FA) y angina estable en la población española $\geq 40$ años. Se trata de un estudio transversal promovido por la Agencia de Investigación de la Sociedad Española de Cardiología y realizado en la población española $\geq 40$ años adscrita a un médico de atención primaria. Su metodología y resultados ya se han descrito en publicaciones previas ${ }^{13,14}$. El médico de atención primaria invitó a los pacientes seleccionados de forma aleatoria (11.831) a participar en el estudio, lo que se logró en un $76 \%$ de los casos. Todos los participantes $(n=8.400)$ otorgaron su consentimiento informado por escrito.

El diagrama de flujo correspondiente a la participación en el estudio OFRECE puede consultarse en publicaciones previas ${ }^{13,14}$. El estudio se inició a nivel nacional en marzo de 2010 y se completó en octubre de 2012. A todos los participantes los examinó su médico de atención primaria, quien completó un formulario de factores de riesgo cardiovascular y antecedentes médicos. Se les realizó un electrocardiograma (ECG) y se midió el peso, la talla y la presión arterial (2 tomas) según las recomendaciones de la Organización Mundial de la Salud, recogidas en las guías vigentes de la Sociedad Europea de Cardiología ${ }^{15}$. Cuando se sospechaba la presencia de una cardiopatía no conocida, se citaba al participante con el cardiólogo coordinador. Las definiciones de los factores de riesgo y antecedentes médicos analizados siguieron los estándares predefinidos del American College of Cardiology/American Heart Association ${ }^{16}$ que están recogidas en el anexo II del material suplementario. Se retiró del análisis a 57 personas por falta de información clave o por ECG no evaluable y se dejó la muestra final en 8.343 personas.

La lectura de los ECG la realizó de forma centralizada un grupo de 9 cardiólogos. Los ECG se asignaron aleatoriamente a cada cardiólogo y se realizó una primera lectura. Posteriormente, y de forma independiente, un segundo cardiólogo revisó todos los trazados. Los casos en los que hubo desacuerdo se pusieron en conocimiento de un tercer observador y se llegó al diagnóstico final por consenso. Los criterios diagnósticos de cada una de las anomalías analizadas fueron los que se describen a continuación.

\section{Intervalo QT-QT corregido}

El intervalo QT absoluto se midió manualmente en las derivaciones II y $\mathrm{V}_{5}$, utilizándose $\mathrm{V}_{4} \mathrm{O}$ $\mathrm{V}_{6}$ o aquella derivación en la que el intervalo estuviera mejor definido cuando no era posible la medición en II o $\mathrm{V}_{5}$. No se consideró la medición automática por 2 motivos: a) no estar disponible en todos los electrocardiógrafos usados en el estudio, y $b$ ) tener diversos algoritmos de medida en aquellos en que sí estaba disponible esta opción. Para obtener el QT corregido (QTc) se utilizó la fórmula de Bazett $(\mathrm{QTc}=\mathrm{QT} / \sqrt{\mathrm{RR}})$. Si bien tiene limitaciones (tendencia a sobreestimar el $\mathrm{QT}$ por 
encima de las 100 pulsaciones), se optó por dicha fórmula por ser la más utilizada. Esto permite comparar los resultados obtenidos con los de otros estudios publicados previamente. Para analizar los intervalos QTc en la población estudiada se establecieron diversas categorías atendiendo a la evidencia clínica disponible, sobre todo en lo que al pronóstico se refiere. Respecto al QTc largo, las categorías fueron las utilizadas en el estudio de Goldenberg et al. ${ }^{17}$ Para el QTc corto se tomaron como referencia las usadas en el artículo de Anttonen et al. ${ }^{18}$ :

- QTc normal: 340-439 ms.

- QTc borderline: 440-469 ms.

- QTc largo: $\geq 470 \mathrm{~ms}^{17,19,20,21}$.

- QTc corto: 321-339 ms.

- QTc muy corto: $\leq 320 \mathrm{~ms}^{11,18,22,23}$.

Se excluyó del análisis a los individuos con bloqueo completo de rama izquierda, ritmos estimulados o ritmos no sinusales.

Con posterioridad a la obtención de resultados se publicaron las nuevas guías de la Sociedad Europea de Cardiología sobre arritmias ventriculares y muerte súbita ${ }^{24}$, que establecen un punto de corte para QTc largo $\geq 480 \mathrm{~ms}$ (clase I, nivel de evidencia C). En consecuencia, se decidió reanalizar los datos referentes a la población del estudio también con este otro punto de corte.

\section{Patrones electrocardiográficos tipo Brugada}

Los patrones electrocardiográficos Brugada 1 y 2 se definieron según el documento de consenso de $2002^{2}$ :

- Tipo 1 o patrón en «lomo de delfín»: elevación convexa-descendente del segmento ST $\geq 2 \mathrm{~mm}$ en más de una derivación precordial derecha, seguida de onda T negativa.

- Tipo 2 o patrón en «silla de montar»: elevación cóncava del segmento ST $\geq 2$ mm en más de una derivación precordial derecha, seguida de ondas $\mathrm{T}$ positivas o isobifásicas.

\section{Análisis estadístico}

Debido al proceso de muestreo, en el que las personas de la población tienen diferentes probabilidades de ser elegidas, a cada participante en la muestra final se le asignó un peso que se interpreta como el número de personas de la población española del mismo grupo de edad, sexo y área geográfica que dicho paciente representa ${ }^{25}$. De este modo, la suma de todos los pesos de la muestra es igual al tamaño de la población española $\geq 40$ años. Esta ponderación fue compleja. Se realizó en 2 fases, de acuerdo a la metodología ya descrita en publicaciones previas del estudio OFRECE $^{13,14}$ y según el procedimiento propuesto por Deville y Särndal ${ }^{26}$ con la instrucción calibrate del paquete estadístico Stata (versión 10.1). Para el ajuste, o calibrado, se utilizó como población el padrón municipal de habitantes del año 2011 por sexo, grupo de edad y área geográfica.

Todos los análisis realizados han tenido en cuenta el diseño muestral del estudio. Para todas las variables analizadas se calcularon las prevalencias específicas por grupos de edad y sexo y sus intervalos de confianza del 95\% (IC95\%).

Para identificar los factores asociados con las anomalías de interés se estimaron las odds ratio ajustadas por edad y sexo mediante modelos de regresión logística. Posteriormente, para identificar factores asociados de manera independiente con las condiciones de interés, se ajustó un modelo multivariante en el que se incluyeron los factores con $\mathrm{p}<0,1$ en el análisis previo. 
Se realizó un análisis de la variabilidad interobservador. La concordancia fue muy alta en todos los casos, superior al 99\%. Respecto a la concordancia en los patrones tipo Brugada, el acuerdo esperado fue 0,9947 y el observado 0,9976. El índice de Kappa fue 0,5444 (IC95\%, 0,36670,7220). En el caso del QTc se anotaron y revisaron los casos en los que la diferencia entre la medición del observador y la del revisor superó los $10 \mathrm{~ms}$ por exceso (la medición del observador es mayor, $n=172$ ) o por defecto (la medición del revisor es mayor, $n=37$ ). Se realizó una tercera medición por ambos y se llegó a un acuerdo. En el resto de los casos (discrepancia $\leq 10 \mathrm{~ms}$ ), se anotó en la base de datos el valor promedio. Estas discrepancias en 209 pares de mediciones (2,5\% del total) tienen un impacto clínico menor puesto que solo 7 (un $0,08 \%$ del total) ocasionarían cambios en la clasificación del paciente en lo que respecta a la duración de su QTc.

\section{RESULTADOS}

\section{Anomalías del QT}

De los 8.343 participantes que completaron el protocolo, 7.889 cumplieron los criterios de inclusión en este análisis (ritmo propio sinusal y no tener bloqueo de rama izquierda). Se excluyó a 243 participantes por estar en FA, a 135 por bloqueo de rama izquierda, a 24 por ambas características y a 52 por presentar ritmos no sinusales o no naturales. El 52,5\% fueron mujeres con una edad media de 58,3 años. La mayor parte de la población (7.011 individuos) tuvo un intervalo QTc normal; lo que supone una tasa del 88,9\%, ponderada por edad y sexo del $90,5 \%$ (IC95\%, 89,5-91,5). El QTc medio en este grupo fue de 403,8 ms (IC95\%, 402,6-405,0). Un total de 763 personas presentó un QTc borderline (prevalencia del 9,7\%; ponderada del 8,3\%; IC95\%, 7,4-9,3). El QTc medio fue 449,3 ms (IC95\%, 448,5-450,0). Presentaron un intervalo QTc largo (punto de corte $\geq 470 \mathrm{~ms}$ ) 96 participantes (prevalencia bruta, 1,22\%; ponderada, 1,01\%; IC95\%, 0,68-1,34). El QTc medio de este grupo de individuos fue de 484,9 ms (IC95\%, 480,2-489,6). Finalmente, 51 participantes tuvieron un QTc $\geq 480 \mathrm{~ms}$, prevalencia del $0,65 \%$ y ponderada del 0,42\% (IC95\%, 0,26-0,57). El QTc medio fue de 499,48 ms (IC95\%, 493,11-505,85). El número de casos con intervalo QTc corto fue de 18 (QTc medio, 333,7 ms; IC95\%, 331,6-335,9), lo que supone una prevalencia del $0,23 \%$ y ponderada por edad y sexo del $0,18 \%$ (IC95\%, 0,08-0,29). No se detectó ningún caso con intervalo QTc muy corto. En la tabla 1 se exponen las características de la población y de los participantes con trastornos del QTc. El análisis multivariante para los trastornos del QTc (tabla 2) mostró que la edad y el antecedente de haber presentado FA se asocian de forma independiente a la presencia de QTc largo (punto de corte $\geq 470 \mathrm{~ms}$ ). La hipertensión arterial mostró una fuerte asociación en el análisis univariante. El análisis multivariante usando el punto de corte $\geq 480 \mathrm{~ms}$ también identificó la FA como factor predictor independiente de QTc largo. Los factores predictores de QTc borderline fueron: edad, ser mujer, hipertensión arterial y diabetes mellitus. Se excluyó del análisis de asociación al grupo de pacientes con QTc corto debido a su pequeño tamaño. 
Tabla 1. Características de la población con QT corregido normal. Comparación población QT corregido normal-QT corregido borderline, QT corregido normal-QT corregido > 470 ms y QT corregido normal-QT corregido > $480 \mathrm{~ms}$

\begin{tabular}{|c|c|c|c|c|c|c|c|c|c|}
\hline & $\begin{array}{c}\text { Normal } \\
(\mathrm{n}=7.011)\end{array}$ & $\begin{array}{c}\text { Borderline } \\
(\mathrm{n}=763)\end{array}$ & $\mathrm{p}$ & $\begin{array}{c}\text { Normal } \\
(\mathrm{n}=7.011)\end{array}$ & $\begin{array}{c}\mathrm{QTc}>470 \\
(\mathrm{n}=96)\end{array}$ & $\mathrm{p}$ & $\begin{array}{c}\text { Normal } \\
(\mathrm{n}=7.011)\end{array}$ & $\begin{array}{c}\mathrm{QTc}>480 \\
(\mathrm{n}=51)\end{array}$ & $\mathrm{p}$ \\
\hline \multicolumn{10}{|l|}{ Sexo $(\%)$} \\
\hline Varones & 48,3 & 38,7 & \multirow{2}{*}{0,002} & 48,3 & 53,1 & \multirow{2}{*}{0,570} & 48,3 & 52,4 & \multirow{2}{*}{0,661} \\
\hline Mujeres & 51,7 & 61,3 & & 51,7 & 46,9 & & 51,7 & 47,6 & \\
\hline \multicolumn{10}{|l|}{ Grupo de edad (\%) } \\
\hline 40-49 años & 33,9 & 21,9 & \multirow{5}{*}{$<0,001$} & 33,9 & 6,3 & \multirow{5}{*}{$<0,001$} & 33,9 & 10,1 & \multirow{5}{*}{$<0,001$} \\
\hline 50-59 años & 25,9 & 24,3 & & 25,9 & 18,5 & & 25,9 & 4,1 & \\
\hline 60-69 años & 19,2 & 19,9 & & 19,2 & 29,3 & & 19,2 & 26,4 & \\
\hline 70-79 años & 13,6 & 17,9 & & 13,6 & 17,6 & & 13,6 & 31,9 & \\
\hline$\geq 80$ años & 7,4 & 16,8 & & 7,4 & 28,4 & & 7,4 & 27,4 & \\
\hline \multicolumn{10}{|l|}{$F R C V$} \\
\hline Obesidad (\%) & 32,4 & 42,4 & 0,002 & 32,4 & 43,9 & 0,029 & 32,4 & 31,4 & 0,892 \\
\hline Sobrepeso $(\%)$ & 42,4 & 39,9 & 0,353 & 42,4 & 31,8 & 0,068 & 42,4 & 28,4 & 0,094 \\
\hline IMC (media, $\left.\mathrm{kg} / \mathrm{m}^{2}\right)$ & 28,3 & 98,4 & 0,003 & 28,3 & 28,9 & 0,311 & 28,3 & 27,2 & 0,294 \\
\hline Hipercolesterolemia (\%) & 23,7 & 30,2 & 0,006 & 23,7 & 30,0 & 0,218 & 23,7 & 28,6 & 0,496 \\
\hline Diabetes $(\%)$ & 10,7 & 19,1 & $<0,001$ & 10,7 & 14,2 & 0,317 & 10,7 & 22,3 & 0,016 \\
\hline Fumador actual (\%) & 23,4 & 21,9 & 0,523 & 23,4 & 12,5 & 0,073 & 23,4 & 9,3 & 0,073 \\
\hline $\operatorname{HTA}(\%)$ & 42,0 & 57,3 & $<0,001$ & 42,0 & 72,1 & $<0,001$ & 42,0 & 63,1 & 0,039 \\
\hline PAS (media, mmHg) & 129,8 & 137,2 & $<0,001$ & 129,8 & 138,4 & $<0,001$ & 129,8 & 136,4 & 0,034 \\
\hline PAD (media, mmHg) & 77,7 & 80,0 & $<0,001$ & 77,7 & 79,9 & 0,138 & 77,7 & 76,5 & 0,404 \\
\hline \multicolumn{10}{|l|}{ Antecedentes (\%) } \\
\hline Accidente cerebrovascular & 3,2 & 3,0 & 0,839 & 3,2 & 8,0 & 0,016 & 3,2 & 6,6 & 0,226 \\
\hline Arteriopatía periférica & 2,1 & 2,7 & 0,331 & 2,1 & 5,2 & 0,052 & 2,1 & 7,6 & 0,017 \\
\hline Enfermedad pulmonar crónica & 5,5 & 7,2 & 0,154 & 5,5 & 14,1 & 0,015 & 5,5 & 26,2 & $<0,001$ \\
\hline Patología tiroidea & 6,7 & 8,6 & 0,226 & 6,7 & 8,2 & 0,638 & 6,7 & 7,8 & 0,777 \\
\hline Implantación de marcapasos/DAI & 0,2 & 0,4 & 0,218 & 0,2 & 7,3 & $<0,001$ & 0,2 & 3,0 & $<0,001$ \\
\hline Cardiopatía isquémica & 4,2 & 3,9 & 0,773 & 4,2 & 8,5 & 0,041 & 4,2 & 14,4 & 0,001 \\
\hline Diagnóstico previo de IC & 1,6 & 3,4 & 0,015 & 1,6 & 5,2 & 0,011 & 1,6 & 3,8 & 0,160 \\
\hline Fibrilación auricular & 1,1 & 1,6 & 0,230 & 1,1 & 6,1 & $<0,001$ & 1,1 & 13,0 & $<0,001$ \\
\hline
\end{tabular}

IC: insuficiencia cardiaca; DAI: desfibrilador automático implantable; FRCV: factores de riesgo cardiovascular; HTA: hipertensión arterial; IMC: índice de masa corporal; PAD: presión arterial diastólica; PAS: presión arterial sistólica; QTc: QT corregido. 
Tabla 2. Análisis multivariante de la población con QT corregido borderline frente a normal y de la población con QT corregido > $470 \mathrm{~ms}$ frente a normal

\begin{tabular}{lccc}
\hline & OR & IC95\% & $\mathrm{p}$ \\
\hline & & & \\
Población con QTc borderline frente a población con QTc normal & & $<0,001$ \\
Edad (años) & 1,02 & $1,01-1,03$ & 0,002 \\
Sexo femenino & 1,48 & $1,16-1,89$ & 0,003 \\
Diabetes mellitus & 1,51 & $1,16-1,98$ & 0,010 \\
Hipertensión arterial & 1,36 & $1,07-1,71$ & $<0,001$ \\
Población con QTc $>470$ ms frente a población con QTc normal & & 0,010 \\
Edad (años) & 1,06 & $1,04-1,09$ & $1,32-9,03$ \\
Fibrilación auricular & 3,46 & & \\
\end{tabular}

IC95\%: intervalo de confianza del 95\%; OR: odds ratio; QTc: QT corregido.

\section{Patrones electrocardiográficos tipo Brugada}

En este análisis se consideraron los datos de los 8.343 individuos del estudio. Se obtuvieron 12 casos de patrones tipo Brugada 1 y 2 :

- Hubo 2 casos de Brugada tipo 1 ( 2 mujeres de 55 y 54 años), lo que supone una prevalencia del $0,024 \%$, ponderada del $0,01 \%$ (IC95\%, 0,00-0,04).

- Fueron 10 los casos de Brugada tipo 2, lo que implica una prevalencia del 0,12\% y ponderada por edad y sexo del $0,17 \%$ (IC95\%, 0,02-0,33). De todos los afectados, 8 eran varones y la edad media del grupo fue de 55,4 años.

En la tabla 3 se muestran las características clínicas de los participantes con patrón electrocardiográfico compatible con Brugada 1 y 2 en comparación con las de la población general. La distribución geográfica de los casos encontrados se muestra en la figura. 
Tabla 3. Características de la población con y sin Brugada (tipos 1 y 2)

\begin{tabular}{|c|c|c|c|c|}
\hline & \multicolumn{3}{|c|}{ Brugada 1 y 2} & \multirow[t]{2}{*}{$\mathrm{p}$} \\
\hline & Todos $(\mathrm{n}=8.343)$ & Sí (n = 12) & No $(\mathrm{n}=8.331)$ & \\
\hline \multicolumn{5}{|l|}{$\operatorname{Sexo}(\%)$} \\
\hline Varones & 47,6 & 50,0 & 47,6 & 0,785 \\
\hline Mujeres & 52,4 & 50,0 & 52,4 & \\
\hline \multicolumn{5}{|l|}{ Grupo de edad (\%) } \\
\hline $40-49$ años & 31,2 & 21,7 & 31,2 & 0,165 \\
\hline 50-59 años & 24,6 & 36,1 & 24,5 & \\
\hline 60-69 años & 19,3 & 5,7 & 19,3 & \\
\hline 70-79 años & 14,9 & 0,0 & 14,9 & \\
\hline$\geq 80$ años & 10,1 & 36,5 & 10,0 & \\
\hline \multicolumn{5}{|l|}{$F R C V$} \\
\hline Obesidad (\%) & 33,8 & 13,2 & 33,9 & 0,092 \\
\hline Sobrepeso $(\%)$ & 42,1 & 69,7 & 42,0 & 0,307 \\
\hline IMC (media, kg/m²) & 28,4 & 27,6 & 28,4 & 0,293 \\
\hline Hipercolesterolemia (\%) & 25,4 & 10,7 & 25,4 & 0,133 \\
\hline Diabetes mellitus (\%) & 12,2 & 0,0 & 12,2 & 0,300 \\
\hline Fumador actual (\%) & 22,3 & 8,7 & 22,4 & 0,630 \\
\hline Hipertensión arterial (\%) & 45,3 & 51,8 & 45,3 & 0,853 \\
\hline PAS (media, mmHg) & 130,9 & 139,1 & 130,8 & 0,424 \\
\hline PAD (media, mmHg) & 77,8 & 76,1 & 77,8 & 0,077 \\
\hline \multicolumn{5}{|l|}{ Antecedentes (\%) } \\
\hline Accidente cerebrovascular & 3,8 & 0,0 & 3,8 & 0,578 \\
\hline Arteriopatía periférica & 2,4 & 0,0 & 2,4 & 0,662 \\
\hline Enfermedad pulmonar crónica & 6,3 & 0,0 & 6,3 & 0,462 \\
\hline Patología tiroidea & 6,9 & 2,8 & 6,9 & 0,254 \\
\hline Implantación de marcapasos/DAI & 0,7 & 0,0 & 0,7 & 0,829 \\
\hline Cardiopatía isquémica & 4,9 & 4,7 & 4,9 & 0,704 \\
\hline Diagnóstico previo de ICC & 3,1 & 0,0 & 3,1 & 0,620 \\
\hline Fibrilación auricular & 4,4 & 0,0 & 4,4 & 0,554 \\
\hline
\end{tabular}

DAI: desfibrilador automático implantable; FRCV: factores de riesgo cardiovascular; ICC: insuficiencia cardiaca congestiva; IMC: índice de masa corporal; PAD: presión arterial diastólica; PAS: presión arterial sistólica. 


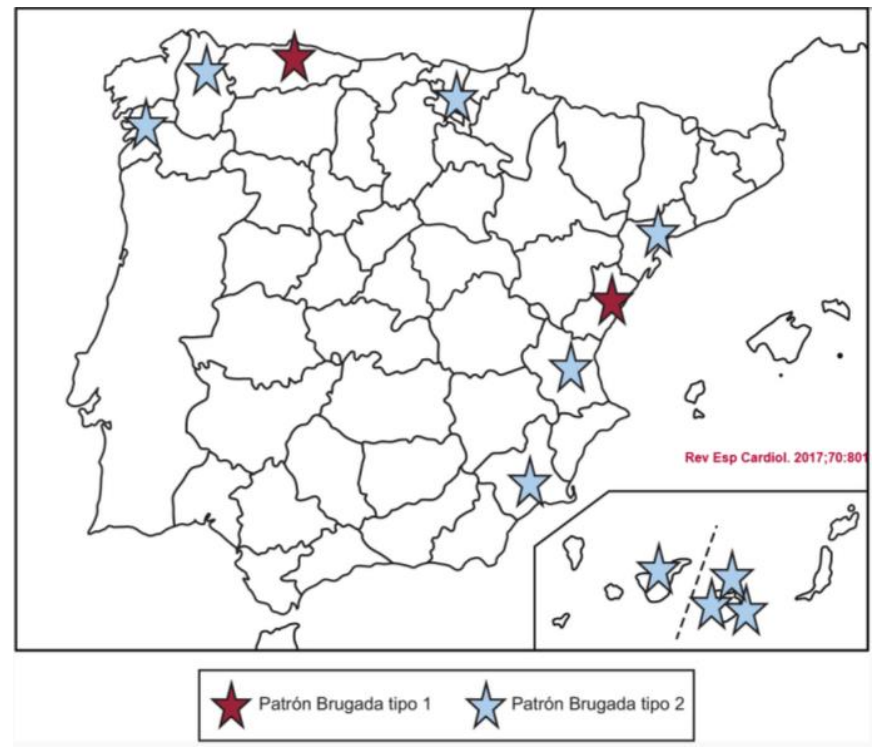

Figura. Distribución geográfica de los patrones electrocardiográficos tipo Brugada encontrados.

\section{DISCUSIÓN}

En este estudio transversal sobre una muestra representativa de la población española de edad $\geq 40$ años se obtiene una prevalencia del 0,01\% de patrón Brugada tipo 1; del 1,01\% de QTc largo con criterio $\geq 470 \mathrm{~ms}$; del $0,42 \%$ con criterio $\geq 480 \mathrm{~ms}$, y del $0,18 \%$ de QTc corto. Por tanto, las anomalías electrocardiográficas asociadas a MS cardiaca están presentes en un porcentaje nada desdeñable de la población general. A la vista de los resultados obtenidos, se podría estimar que entre el 0,6 y el 1,2\% de la población $\geq 40$ años tiene un patrón electrocardiográfico asociado a mayor riesgo de presentar MS. Si además se considerase a las personas con QTc borderline y con patrón Brugada tipo 2, este porcentaje sería cercano al $10 \%$.

\section{Patrones asociados al síndrome de Brugada}

La prevalencia encontrada de los patrones tipo Brugada 1 y 2 fue baja $(0,13 \%)$, dato que está en consonancia con las series europeas publicadas después de 2002 en Grecia, Italia y Turquía ${ }^{27,28,29}$. La prevalencia en países del norte (Alemania, Finlandia y Dinamarca) ${ }^{10,30,31}$ fue baja y no se documentó ningún patrón de Brugada tipo 1. En el presente estudio llama la atención que los 2 casos de Brugada tipo 1 identificados corresponden a 2 mujeres de mediana edad ya que clásicamente se considera que el síndrome de Brugada afecta con mayor frecuencia a los varones, con una proporción de $8: 1^{2}$. En ese sentido, los hallazgos de los casos de patrón Brugada tipo 2 son más concordantes con la literatura médica (de 10 casos descritos, 8 son varones). En este estudio, la prevalencia encontrada viene determinada por incluir únicamente a individuos mayores de 40 años. Sin embargo, desde el punto de vista pronóstico, cabe la posibilidad de que este sesgo de selección tenga una importancia relativa; ya que esas mismas revisiones cifran en 40 años la edad media de inicio o diagnóstico de los pacientes afectados. 


\section{Anomalías del QT}

La prevalencia de anormalidades del QT ha sido alta. Si se toma como referencia el trabajo de Goldenberg et al. ${ }^{17}$ se puede decir que hay evidencias de que el QTc borderline o mayor de $470 \mathrm{~ms}$ se asocia a mayor riesgo de presentar eventos cardiacos. Las prevalencias encontradas en esta serie son altas: un $8,3 \%$ y un $1,01 \%$ ( $\geq 470 \mathrm{~ms}$ ) respectivamente. Esto permite estimar que en España hay casi 4 millones de personas que presentan algún grado de prolongación del intervalo QTc, con el consiguiente riesgo de presentar eventos cardiacos. No obstante, otros estudios han mostrado prevalencias todavía más altas. En el análisis de Veglio et al. ${ }^{20}$ se documentó una prevalencia bruta del $25,8 \%$ de individuos con QTc > $440 \mathrm{~ms}$. Se trataba de una cohorte pequeña y seleccionada: todos los integrantes tenían diabetes tipo 2 , una edad media de 65,4 años y predominaba el sexo femenino. Si se tiene en cuenta que el alargamiento del QTc se relacionó significativamente con la edad, el sexo femenino y el diagnóstico de diabetes, esto por sí solo justifica que se observaran prevalencias mayores que en el presente estudio. Los resultados del Rotterdam Study ${ }^{21}$ también muestran prevalencias mucho más altas que las obtenidas en el actual estudio: un $18 \%$ de QTc borderline y un 11,1\% de QTc largo. Una vez más, la edad media de la población analizada es más alta y hay más mujeres que varones. Además, los puntos de corte utilizados son diferentes de los obtenidos en nuestro estudio: mucho más restrictivos. Así, estos factores pueden haber contribuido a las diferencias encontradas entre ambos análisis. En nuestro estudio, y en lo que al perfil de pacientes afectados se refiere, el grupo con QTc largo muestra un patrón clínico diferente al del grupo con QTc borderline. El QTc borderline identifica a individuos con alta carga de riesgo cardiovascular, ya que factores como la diabetes y la hipertensión arterial se constituyeron como predictores independientes. La carga de riesgo cardiovascular no influyó a la hora de presentar QTc largo. En este caso, el alargamiento del QTc por encima de 470 o $480 \mathrm{~ms}$ tuvo una fuerte asociación con haber presentado episodios previos de FA. Con respecto al papel que pueda desempeñar el uso de fármacos antiarrítmicos en este contexto, lo cierto es que tan solo 2 pacientes de los que tenían antecedentes de FA estaban en tratamiento con amiodarona o un fármaco de clase IC. Por otro lado, se desconoce el número de participantes que podrían haber estado recibiendo estos fármacos como tratamiento de otras arritmias. En consecuencia, no es posible establecer con exactitud el porcentaje de individuos en tratamiento antiarrítmico que podría justificar la presencia de QTc largo. Al margen de un posible efecto farmacológico, cabe la posibilidad de que exista una característica no identificada en estos individuos que condicione el alargamiento del QT (quizá factores genéticos relacionados con el síndrome de QT largo congénito). Estos factores también podrían predisponer a los portadores a presentar FA. Al igual que en estudios previos, el QTc borderline se relacionó significativamente con la edad y el sexo femenino ${ }^{17,20,21}$. No fue así en el QTc largo, cuya presencia no dependió del sexo aunque sí de la edad.

Respecto al QTc corto, la prevalencia obtenida ha sido de un $0,23 \%$ - algo más baja que las prevalencias publicadas en las series japonesas ${ }^{32,33}$ y más en la línea de las europeas ${ }^{11,18}$-, lo que se debe al punto de corte utilizado en este caso (340 ms o menos).

A la vista de los resultados, parece claro que es necesario obtener más información acerca del verdadero pronóstico de estos hallazgos. Un estudio de seguimiento en estas poblaciones permitiría identificar qué grupo de pacientes se beneficiaría de un seguimiento clínico más específico.

\section{Limitaciones}

\section{Relacionadas con la selección de la muestra}

Aunque el diseño transversal con selección aleatoria de participantes entre la población general no es un diseño eficiente en condiciones de muy baja prevalencia (especialmente en el caso del síndrome de Brugada), ha sido posible llevarlo a cabo gracias a la realización del proyecto OFRECE. Como se ha indicado con anterioridad, este estudio proporciona garantías razonables de representar adecuadamente a la población española $\geq 40$ años $^{13}$. La limitación de ser un estudio transversal y para la población de 40 años o más es inevitable. Es probable que, en condiciones relacionadas con la mortalidad precoz (como el síndrome de Brugada), se infravalore la prevalencia real por el hecho de incluir solo a personas que 
sobreviven hasta los 40 años. La lectura es que, muy probablemente, la magnitud del problema que se presenta es una estimación a la baja de la real.

\title{
Relacionadas con la realización e interpretación del electrocardiograma
}

Otra limitación es la relacionada con la técnica de adquisición de los trazados. Se sabe que es fundamental realizar los ECG de acuerdo con unos determinados estándares. La aplicación correcta de los filtros de paso alto y paso bajo, la velocidad del papel, el voltaje, etc. son factores que no se han podido controlar y se han tenido que analizar ECG realizados con diferentes electrocardiógrafos. Este hecho ha podido influir en algunos diagnósticos, pero hacerlo de manera más rigurosa hubiera sido tan complejo y costoso que habría amenazado la viabilidad del estudio. No obstante, estas limitaciones técnicas, tienen la ventaja de que acercan el estudio a la práctica clínica real. Parte de estas limitaciones se han podido corregir al haberse hecho el análisis de los ECG de forma centralizada.

El carácter intermitente de algunos trastornos constituye otra limitación; la prevalencia del patrón Brugada puede estar infraestimada, ya que, en ocasiones, este únicamente se pone de manifiesto en determinadas circunstancias (fiebre o uso de algunos fármacos). Respecto al QT largo, no se ha podido establecer qué pacientes presentaban un alargamiento del QT de origen farmacológico y qué porcentaje podría corresponder a una alteración congénita. La información relativa al uso de fármacos en este grupo de pacientes es limitada y no hay datos disponibles en cuanto al uso de antihipertensivos, antibióticos, antiepilépticos u otros fármacos implicados en alargamiento del QT: solo se dispuso de información referente a los fármacos usados en la FA. En consecuencia, no es posible saber quiénes son los pacientes con QT largo sin que medie fármaco alguno.

\section{CONCLUSIONES}

Los patrones electrocardiográficos asociados a MS de origen cardiaco son frecuentes en la población española de 40 años o más: entre un 0,6 y un 1,1\% presenta alguna anomalía electrocardiográfica asociada con mayor riesgo de MS por arritmias ventriculares. Esto supone que en España hay unas 480.000 personas en esta situación. El intervalo QTc largo ha sido el hallazgo más frecuente (entre el 0,42 y el $1 \%$ de los casos según el criterio utilizado) y se ha asociado con historia previa de FA y con mayor edad. El QTc borderline - también asociado a MS cardiaca, aunque en menor grado- mostró una prevalencia alta (8,3\%), alrededor de 3.332 .000 personas de edad $\geq 40$ años en España. Se asoció a mayor edad, sexo femenino y a factores de riesgo cardiovascular (hipertensión arterial y diabetes). El patrón Brugada tipo 1 mostró muy baja prevalencia $(0,01 \%)$, semejante a la descrita en otras series de nuestro entorno.

\section{FINANCIACIÓN}

El promotor del estudio OFRECE es la Agencia de Investigación de la Sociedad Española de Cardiología. Este estudio se ha financiado con una beca del Observatorio de la Mujer de la Agencia de Calidad del Ministerio de Sanidad y con una beca sin condiciones de SANOFI (que no participó en el diseño del estudio, el análisis de datos o la preparación del manuscrito).

\section{CONFLICTO DE INTERESES}

\author{
Ninguno.
}




\section{MATERIAL SUPLEMENTARIO}

Se puede consultar material suplementario a este artículo en su versión electrónica disponible en http://dx.doi.org/ 10.1016/j.recesp.2016.11.029.

\section{BIBLIOGRAFÍA}

1. Brugada P, Brugada J. Right bundle branch block, persistent ST segment elevation and sudden cardiac death: A distinct clinical and electrocardiographic syndrome: A multicenter report. J Am Coll Cardiol. 1992;20:1391-6.

2. Wilde AA, Antzelevitch C, Borggrefe M, et al. Proposed diagnostic criteria for the Brugada syndrome: consensus report. Circulation. 2002;106:2514-9.

3. Antzelevitch C, Brugada P, Borggrefe M, et al. Brugada syndrome. Report of the second consensus conference. Circulation. 2005;111:659-70.

4. Antzelevitch C. Brugada syndrome. Pacing Clin Electrophysiol. 2006;29:1130-59.

5. Gussak I, Brugada P, Brugada J, et al. Idiopathic short QT interval a new clinical syndrome?. Cardiology. 2000;94:99-102.

6. Gaita F, Giustetto C, Bianchi F, et al. Short QT syndrome: a familial cause of sudden death. Circulation. 2003;108:965-70.

7. Medeiros-Domingo A, Iturralde-Torres P, Ackerman MJ. Clínica y genética en el síndrome de QT largo. Rev Esp Cardiol. 2007;60:739-52.

8. Van Noord C, Eijgelsheim M, Stricker BH. Drug and non-drug-associated QT interval prolongation. Br J Clin Pharmacol. 2010;70:16-23.

9. Hermida JS, Lemoine JL, Aoun FB, et al. Prevalence of the brugada syndrome in an apparently healthy population. Am J Cardiol. 2000;86:91-4.

10. Sinner MF, Pfeufer A, Perz S, et al. Spontaneous Brugada electrocardiogram patterns are rare in the German general population: results from the KORA study. Europace. 2009;11:1338-44.

11. Bjerregaard P, Nallapaneni H, Gussak I. Short QT interval in clinical practice. J Electrocardiol. 2010;43:390-5.

12. Hocini M, Pison L, Proclemer A, et al. Scientific Initiative Committee, European Heart Rhythm Association. Diagnosis and management of patients with inherited arrhythmia syndromes in Europe: results of the European Heart Rhythm Association Survey. Europace. 2014;16:600-3.

13. Gómez-Doblas JJ, Muñiz J, Alonso Martin JJ, et al. Prevalencia de fibrilación auricular en España. Resultados del estudio OFRECE. Rev Esp Cardiol. 2014;67:259-69.

14. Alonso JJ, Muñiz J, Gómez-Doblas JJ, et al. Prevalencia de angina estable en España. Resultados del estudio OFRECE. Rev Esp Cardiol. 2015;68:691-9.

15. Mancia G, De Backer G, Dominiczak A, et al. Guidelines for the management of arterial hypertension The Task Force for the Management of Arterial Hypertension of the European Society of Hypertension (ESH) and of the European Society of Cardiology (ESC). Eur Heart J. 2007;28:1462-536.

16. McNamara RL, Brass LM, Drozda JP, et al. ACC/AHA key data elements and definitions for measuring the clinical management and outcomes of patients with atrial fibrillation. A report of the American College of Cardiology/American Heart Association Task Force on Clinical Data Standards (Writing Committee to Develop Data Standards on atrial Fibrillation). J Am Coll Cardiol. 2004;44:475-95.

17. Goldenberg I, Moss AJ, Bradley J, et al. Long-QT syndrome after age 40. Circulation. 2008;117:2192201.

18. Anttonen O, Junttila MJ, Rissanen H, et al. Prevalence and prognostic significance of short QT interval in a middle-aged Finnish population. Circulation. 2007;116:714-20.

19. Schwartz PJ, Stramba-Badiale M, Crotti L, et al. Prevalence of the congenital long-QT syndrome. Circulation. 2009;120:1761-7.

20. Veglio M, Bruno G, Borra M, et al. Prevalence of increased QT interval duration and dispersion in type 2 diabetic patients and its relationship with coronary heart disease: a population-based cohort. $J$ Intern Med. 2002;251:317-24.

21. Straus SM, Kors JA, De Bruin ML, et al. Prolonged QTc interval and risk of sudden cardiac death in a population of older adults. J Am Coll Cardiol. 2006;47:362-7.

22. Gollob MH, Redpath CJ, Roberts JD. The Short QT Syndrome. Proposed Diagnostic Criteria. J Am Coll Cardiol. 2011;57:802-12.

23. Bjerregaard P. Proposed Diagnostic Criteria for Short QT Syndrome Are Badly Founded. J Am Coll Cardiol. 2011;58:549-50. 
24. Priori SG, Blomstrom-Lundqvist C, Mazzanti A, et al. 2015 ESC Guidelines for the management of patients with ventricular arrhythmias and the prevention of sudden cardiac death: The Task Force for the Management of Patients with Ventricular Arrhythmias and the Prevention of Sudden Cardiac Death of the European Society of Cardiology (ESC). Endorsed by: Association for European Paediatric and Congenital Cardiology (AEPC). Eur Heart J. 2015;36:2793-867.

25. Silva LC. Diseño razonado de muestras y captación de datos para la investigación sanitaria. Madrid: Díaz de Santos;2000.

26. Deville JC, Särndal CE. Calibration estimators in survey sampling. J Am Statist Assoc. 1992;87:376-82.

27. Letsas KP, Gavrielatos G, Efremidis M, et al. Prevalence of Brugada sign in a Greek tertiary hospital population. Europace. 2007;9:1077-80.

28. Gallagher MM, Forleo GB, Behr ER, et al. Prevalence and significance of Brugada-type ECG in 12,012 apparently healthy European subjects. Int J Cardiol. 2007;130:44-8.

29. Bozkurt A, Yas D, Seydaoglu G, et al. Frequency of Brugada-type ECG pattern (Brugada sign) in Southern Turkey. Int Heart J. 2006;47:541-7.

30. Junttila MJ, Raatikainen MJ, Karjalainen J, et al. Prevalence and prognosis of subjects with Brugada-type ECG pattern in a young and middle-aged Finnish population. Eur Heart J. 2004;25:874-8.

31. Pecini R, Cedergreen $P$, Theilade $S$, et al. The prevalence and relevance of the Brugada-type electrocardiogram in the Danish general population: data from the Copenhagen City Heart Study. Europace. 2010;12:982-6.

32. Funada A, Hayashi K, Ino H, et al. Assessment of QT Intervals and Prevalence of Short QT Syndrome in Japan. Clin Cardiol. 2008;31:270-4.

33. Miyamoto A, Hayashi H, Yoshino T, et al. Clinical and electrocardiographic characteristics of patients with short QT interval in a large hospital-based population. Heart Rhythm. 2012;9:66-74. 